\title{
LES ÉTUDES DE PALÉOGRAPHIE DES ÉCRITURES LIVRESQUES ARABES: QUELQUES OBSERVATIONS
}

\author{
François DÉROCHE \\ École Pratique des Hautes Études, París \\ Algo se ha hecho, pero poco \\ F. Codera, "Paleografía árabe», \\ Boletín de la Real Academia \\ de la Historia, 33, 1898.
}

Il y a de cela un siècle, F. Codera dressait à propos de la paléographie arabe le constat désabusé que nous citons en épigraphe. Animé cependant par la ferme conviction qu'il était possible de créer —ce sont ses mots-cette discipline, il traçait un programme ambitieux et remarquable en ce qu'il anticipait en partie le chemin que devait suivre la paléographie latine au cours du $\mathrm{XX}^{\mathrm{e}}$ siècle.

Qu'en a-t-il été de la paléographie des écritures livresques arabes? Une anecdote que nous avions lue alors que nous venions de commencer l'étude du fonds des manuscrits du Coran conservés à la Biblitohèque Nationale de France nous avait laissé entrevoir à quel point les études en ce domaine demeuraient enveloppées d'un voile de mystère. C'était en effet presque une scène initiatique que rapportait $\mathrm{P}$. Sj. Van Koningsveld ${ }^{1}$ dans les institutions possédant un fonds de manuscrits arabes, les étudiants avancés, préparant sans doute l'édition d'un texte, avaient coutume selon lui de tester les débutants en leur présentant un manuscrit ouvert dont ils tenaient le colophon ou le début dissimulés; le jeu consistait à leur demander de dater, puis de localiser la copie. Si leur réponse était satisfaisante, ils entraient dans le cercle des happy few qui s'y entendaient en manuscrits arabes... Comment, pensions-nous, pouvait-on répondre à une question de ce genre alors qu'il n'existait pas de manuel de paléographie, à peine quelques bribes d'enseignement et si peu d'albums où pouvoir acquérir cette expérience des écritures livresques?

La paléographie, science des écritures anciennes, se penche sur des documents qui ont été portés sur des supports divers (parchemin, pierre, métal, papier, etc.) au moyen d'utensiles variés (ciseau à bois, calame, pinceau, ongles, etc.); du fait des particularités qui découlent de chacune de ces situations, des observations paléographiques plus spécifiques à tel ou tel type de document comme les inscriptions, les monnaies ou les papyrus, ont été proposées dans le cadre de disciplines spécialisées — l'épigraphie, la numismatique ou la papyrologie—qui

\footnotetext{
${ }^{1}$ The Latin-Arabic glossary of the Leiden University Library, Leiden, 1976, p. 25.
} 
couvrent une partie du champ que se propose la paléographie. La situation du livre manuscrit n'est en rien différente: la discipline qui le prend pour objet d'étude, la codicologie, englobe la paléographie en tant qu'elle s'applique à l'écriture des manuscrits et lui apporte le soutien des autres savoirs spécialisés que le codicologue met en œuvre ${ }^{2}$.

La paléographie, apparue il y a trois cents ans, a traditionnellement pour but le déchiffrement correct des écritures anciennes. Elle doit également permettre de dater ces dernières et, dans une phase ultérieure de son développement, elle peut permettre de proposer une histoire des écritures qu'elle a prises pour objet. À la base de la démarche paléographique se trouve la comparaison: de manière très schématique, on admet qu'une copie A, non datée mais présentant une étroite ressemblance avec le groupe graphiquement cohérent $B$, a dû être copiée vers la même époque que les manuscrits de B -lesquels sont datés et éventuellement localisés. La réalité est naturellement plus complexe, mais cette façon très simplifiée d'exposer les choses nous amène à souligner d'une part l'importance des documents eux-mêmes, et de l'autre celle de ces séries qui constituent l'amorce d'une typologie.

L'existence d'une terminologie qui caractérise les différentes variétés de l'écriture arabe pourrait a priori être perçue comme l'indice que cette typologie existe bel et bien. Or l'avis de certains spécialistes des manuscrits trahit de fortes réticences à propos de la classification des écritures telle qu'elle est actuellement pratiquée. Dans une livraison de l'Encyclopédie de l'Islam publiée en 1980, on pouvait lire sous la plume d'A. D. H. Bivar: «Des manuels arabes de calligraphie, suivis par plusieurs commentateurs récents, appliquent des termes spéciaux

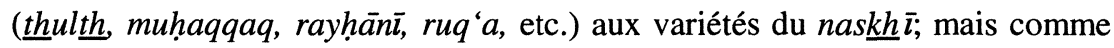
l'application de ces termes à des périodes successives ne rime à rien et que les catégories qui figurent sur les monuments n'y correspondent pas toujours, il est plus sûr de n'employer que le terme général ${ }^{3}$." Dans la postface à un album paléographique, J. J. Witkam avait écrit deux ans plus tôt ${ }^{4}$ : "En dehors de quelques remarques sur les particularités les plus évidentes des écritures [reproduites dans l'album où figurent ces lignes], j'ai dû m'abstenir de donner une

\footnotetext{
${ }^{2} \mathrm{La}$ question des rapports entre paléographie et codicologie est loin de faire l'unanimité: pour certains, la codicologie est un appoint, pour d'autres elle réunit les différentes approches du livre manuscrit. On pourra se reporter, à titre d'exemple, à ce qu'écrivent A. Dain (Les manuscrits, Paris, 1964, p. 77) et J. Lemaire (Introduction à la codicologie [Publ. de l'Institut d'Études Médiévales: Textes, Études, Congrès 9], Louvain-la-Neuve, 1989, p. 3).

${ }^{3} E l^{2}$, art. kitabät (éd. française, t. V, p. 228).

${ }^{4}$ Seven specimens of Arabic manuscripts preserved in the Library of the University of Leiden, Leiden, 1978, p. 18.
} 
analyse paléographique de ces spécimens. Cela tient au fait que, dans ce domaine, même le travail le plus élémentaire reste encore à faire. Aucun critère adapté à la description de l'écriture arabe n'existe encore. Le simple fait que toutes les écritures présentées ici puissent commodément être appelées naskhi prouve que ce nom est à peine utilisable et pourrait tout aussi bien être abandonné.» Quoique partiellement divergentes, les remarques critiques de ces deux savants permettent de constater que la nomenclature —c'est à dire in fine la typologie-des écritures arabes, telle qu'elle existe, est loin de satisfaire aux exigences scientifiques.

Comment expliquer que, depuis l'époque de F. Codera, si peu d'avancées substantielles aient été réalisées? Le nombre des documents à prendre en considération, le fait qu'ils demeurent souvent difficilement accessibles ralentissent sans aucun doute les progrès de la recherche. Il faut pourtant, selon nous, remonter à une époque plus ancienne pour appréhender les origines des difficultés qui ont fait obstacle au développement de la paléographie des écritures arabes livresques. Le modèle latin ou grec a sans aucun doute fortement influencé les arabisants, quand, vers la fin du XVIII ${ }^{\mathrm{e}}$ siècle, ils commencèrent à leur tour à parler d'une paléographie arabe ${ }^{5}$. Les circonstances dans lesquelles ils se trouvaient différaient cependant très fortement de celles qu'avaient connues un Mabillon ou un Montfaucon, tout particulièrement en ce qui concerne le livre manuscrit.

En effet, alors que l'invention de Gutenberg avait sonné le glas de la copie des manuscrits en Europe, la production manuscrite restait extrêmement vivante et abondante dans le monde islamique. Une véritable culture de l'écriture lui était étroitement associée: elle constitue un aspect spécifique de la civilisation islamique et réunissait entre autres à l'époque des éléments d'une histoire de l'écriture arabe ${ }^{6}$, une nomenclature des différentes graphies ${ }^{7}$ et un ensemble de savoir traditionnels, soit de nature technique, soit de l'ordre des us et coutumes. De ce fait, les orientalistes pouvaient trouver, soit dans les sources écrites, soit auprès de professionnels du monde musulman des réponses inmédiates aux questions qu'ils se posaient au sujet de l'écriture. Une anecdote illustre parfaitement cette situation: Étienne Legrand, un orientaliste français qui séjournait au Caire vers le milieu du XVIII ${ }^{e}$ siècle, souhaitait déchiffrer les inscriptions anciennes qu'il voyait

\footnotetext{
${ }^{5}$ Le mot apparaît semble-t-il pour la première fois dans le contexte des études arabes sous la plume de J. G. C. Adler (Museum cuficum Borgianum Velitris. Rome, 1782, p. 32).

${ }^{6}$ Les premiers exemples datent du $\mathrm{III}^{\mathrm{e}} / \mathrm{IX}^{\mathrm{e}}$ siècle (par ex. chez al-Bagdādī; $c f$. Sourdel, D., "Le 'Livre des secrétaires' de 'Abdallāh al-Baġdādī', BEO 14 [1952-54], 128-130); à l'époque ottomane, des traités comme Hatt ve-hatțātān de Habīb Ef. (Constantinople, 1305/1887-88) comportent des indications - brèves - sur ce sujet.

${ }^{7}$ On se reportera à Abbot, N., "Arabic paleography", Ars islamica 8 (1941), 79 et suiv.
} 
sur les monuments de la ville. Il réussit à trouver un savant local qui, moyennant rétribution, accepta d'écrire un ouvrage où ces questions seraient traitées; bien que l'Egyptien n'ait pas terminé son travail, il avait quand même remis à son commanditaire 410 pages in $-4^{\circ}$ sur la question $!^{8}$

Cette conviction, en partie fondée, que les sources orientales étaient à même de procurer les éléments d'une paléographie arabe en bonne et due forme a pesé lourdement sur le développement de la discipline. En effet, l'effort de ceux qui se sont penchés sur ces questions s'est porté en priorité sur l'exploitation méticuleuse des sources - sans avoir pris la mesure des hypothèques qui grevaient ce savoir traditionnel, quelque estimable et important qu'il puisse être; de ce fait, les documents eux-mêmes se trouvèrent relégués au second plan ${ }^{9}$. Il convient toutefois d'ajouter qu'à la fin du XVIII ${ }^{e}$ s. les collections de manuscrits dont pouvaient effectivement disposer les arabisants européens restaient de taille bien modeste et n'étaient pas toujours représentatives de la diversité graphique du monde islamique: cela a sans nul doute contribué à limiter la place des documents dans les premiers travaux paléographiques.

Revenons un instant aux sources orientales: leur évaluation critique, préalable à toute utilisation, n'a jamais été menée à bien de manière systématique. Que l'on pense, par exemple, au mä' $i l$, cette graphie dont le nom figurait dans le texte du Fihrist publié par G. Flügel et que J. von Karabacek pensait reconnaître sur les feuillets du ms Londres, BL Or. $2165^{10}$. Une édition plus récente du texte, fondée sur des copies de l'œuvre plus anciennes que celles utilisées par G. Flügel, révèle que l'auteur mentionnait à cet endroit une écriture appelée munābid ${ }^{11}$. Le mot $m \bar{a}$ ' $l$ devrait donc disparaître de la littérature scientifique et la critique textuelle pourrait à l'avenir imposer d'autres révisions analogues...

Il y a plus: la culture calligraphique musulmane a préservé à travers les siècles une terminologie ancienne qui a pu soit être appliquée à des réalités graphiques changeantes au fil des générations ou des régions, soit perdre tout rapport avec

\footnotetext{
${ }^{8}$ Silvestre de Sacy, A. I., «Mémoire sur l'origine et les anciens monuments de l'écriture parmi les Arabes", Mémoires de littérature, tirés des registres de l'Académie... des inscriptions et belles-lettres 50, 1808, p. 265 (reédité dans Déroche, F., et Noja Noseda, S., Le manuscrit arabe 328(a) de la Bibliothèque nationale de France [Sources de la transmission manuscrite du texte coranique 1/1]. Lesa, 1998, p. XXXV).

${ }^{9} \mathrm{P}$. Sj. van Koningsveld (op. cit., p. 70, n. 94) a pris la mesure du problème que pose cette approche des questions paléographiques et se proposait de partir des documents eux-mêmes.

${ }^{10}$ Von Karabacek, J., «Julius Euting's Sinaitische Inschriften», WZKM 5 (1891), 323-324 (d'après Ibn al-Nadīm, K. al-Fihrist, éd. G. Flügel, Leipzig, 1871).

${ }^{11}$ Ibn al-Nadìm, K. al-Fihrist, éd. Tajaddud. Téhéran, 1350/1971; cette lecture est celle qu'a suivie Bayard Dodge dans sa traduction (The Fihrist of al-Nadim, A tenth century survey of Muslim culture I. New-York/London, 1970, p. 11).
} 
une écriture identifiable par ceux qui transmettaient le mot $^{12}$. Cette situation n'est nullement unique: la nomenclature des textiles ${ }^{13}$, ou encore celle de techniques comme l'archerie a conservé fidèlement des noms dont la signification exacte s'était perdue à un moment ou à un autre ${ }^{14}$. En fait, cette culture calligraphique si caractéristique du monde islamique n'avait nullement vocation à servir de paléographie: elle jouait un rôle important au sein d'un système culturel complexe qui valorisait fortement l'écriture, mais ne visait ni à apprendre à déchiffrer des graphies anciennes ${ }^{15}$, ni à dater les manuscrits ${ }^{16}$. Elle pouvait fournir des éléments d'appréciation, parfois très fins, dans un cadre chronologique et géographique précis; la tradition ottomane illustre à merveille cet état de choses: encore aujourd'hui, ses représentants savent déceler les menues particularités du style de tel ou tel calligraphe appartenant à cette lignée, mais paraissent bien moins à l'aise devant des œuvres produites en dehors d'elle.

Il serait cependant excessif de conclure que la typologie des écritures empruntée à la tradition orientale est totalement inopérante. Elle possède au contraire une certaine validité, comme le montre l'expérience courante: si dans une description de manuscrit apparaissent les mots magrib $\bar{\imath}$ ou nasta'li $q$ nous saisissons immédiatement de quoi il s'agit. Dans le même temps —et ce tant que

${ }^{12}$ L'approche encyclopédique et cumulative traditionnelle du savoir a pour effet de brouiller les cartes en mêlant des notions anciennes avec d'autres, contemporaines de l'auteur; cette façon de faire est naturellement difficilement compatible avec la nature historique de la paléographie. Elle perdure néanmoins comme le montre le catalogue d'une exposition tenue à Riyadh où une liste de 137 noms d'écritures est introduite par la remarque suivante: "Most of the names <found in historical and literary reference texts $>$ have been compiled and listed according to their meanings. And to this extensive list of historical names the most recent names have also been added" (Arabic calligraphy in manuscripts [An exhibition on Arabic calligraphy held at the Islamic Art Gallery of the King Faisal Center for Research and Islamic Studies]. Riyadh, 1406/1986, p. 26).

${ }^{13}$ On se reportera à l'opinion exprimée à ce sujet par Cornu, G., Tissus d'Egypte, Témoins du monde arabe, Collection Bouvier. Genève-Paris, 1993, p. 28: “On ne peut qu'avoir recours à la méthode des comparaisons, à partir de pièces datées et localisées de façon exacte grâce à des inscriptions... Mais le plus difficile est l'identification de ces textiles dans le cadre du vocabulaire arabe de l'époque correspondante. Les textes ne donnent jamais de description assez détaillée pour que l'on soit sûr que tel terme s'applique à tel ou tel échantillon de collection. On en est réduit aux hypothèses."

${ }^{14}$ Voir Bivar, A. D. H., c. r. de Safadi, Y. H., "Islamic calligraphy", BSOAS 42 (1979), 564.

15 À la différence des manuscrits latins médiévaux, les manuscrits arabes n'ont pas recours dans la copie du texte même à de multiples abréviations. De ce fait, la part de connaissances techniques requise pour la lecture des manuscrits est relativement limitée. En revanche, les savants musulmans étaient tout à fait conscients des difficultés de lecture inhérentes à l'écriture arabe elle-même ( $c f$. Rosenthal, F., The technique and approach of Muslim scholarship [Analecta orientalia 24], Rome, 1947, p. 24-26).

${ }^{16}$ Comme le signale F. Rosenthal, les savants du monde islamique médiéval se limitaient à des appréciations très vagues —comme "ancien", "très ancien" ou "de l'époque de l'auteur"— lorsqu'ils avaient à apprécier l'âge d'une copie non datée (op. cit., p. 23, n. 3). Des colophons peuvent parfois comporter des indications qui reflètent les connaissances "paléographiques" médiévales (voir par ex. Fi.M.M.O.D. 30: ms Paris, BnF Arabe 167); il serait intéressant d'exploiter cette source d'information. 
nous n'aurons pas vu le manuscrit lui-même, nous restons conscients des variétés considérables que recouvre chacun des deux termes évoqués. Il n'empêche: cette capacité relative à décrire les écritures n'a pu que conforter l'usage de la terminologie traditionnelle.

Ce quiproquo a perduré, la paléographie arabe se contentant d'ajuster son cadre historique aux évidences contraignantes: ainsi, lorsque les premiers papyrus parviennent entre les mains des arabisants, le coufique perd son antériorité absolue $^{17}$. Parfois aussi, des voix comme celle de F. Codera, s'élèvent pour appeler à l'élaboration d'une paléographie des écritures arabes livresques qui, pour remplir pleinement ses fonctions, parte des manuscrits eux-mêmes ${ }^{18}$. Mais la tendance dominante reste celle d'une exégèse toujours plus fine des sources arabes, laquelle trouve son expression la plus achevée avec les travaux de $\mathrm{N}$. Abbott: dans son livre The Rise of the North Arabic script, elle met une érudition remarquable au service d'une conception «nominaliste» de l'histoire de l'écriture ${ }^{19}$.

Dans certains domaines comme l'épigraphie, la paléographie arabe évolua toutefois de façon différente. Les travaux d'un Max van Berchem ou encore d'un Samuel Flury, serrant de plus près la réalité des inscriptions, progressèrent vers une typologie plus fine des écritures des premiers siècles de l'hégire. La spécificité de l'épigraphie arabe empêche malheureusement cette dernière de tenir le rôle de guide pour ceux qui s'occupent de manuscrits; dans une certaine mesure, elle a même pu accentuer le poids d'une approche qui, en accordant une attention soutenue aux éléments décoratifs, se place davantage dans la perspective de l'histoire de l'art que dans celle de la paléographie stricto $s e n s u^{20}$.

\footnotetext{
${ }^{17}$ Voir Silvestre de Sacy, A. I., «Lettre au rédacteur sur d'anciens manuscrits sur papyrus écrits en caractères arabes neskhi» Journal asiatique 7 (1825); puis du même, "Mémoire sur quelques papyrus écrits en arabe et récemment trouvés en Egypte», Mémoires de l'Académie des inscriptions et belleslettres 9, 1831. Le "coufique» continue cependant à apparaître parfois comme l'écriture arabe la plus ancienne: dans un article paru en 1991, Y. Tabbaa le fait figurer à l'origine de l'arbre généalogique des écritures ("The transformation of Arabic writing, Part 1, Qur'ānic calligraphy», Ars Orientalis 24 (1991), 123), en spécifiant que le mot était employé dans son sens originel «as the mother script of all later Arabic scripts» (?). Cette afirmation parait pour le moins aventurée, si l'on en juge par le texte d'Ibn al-Nadīm.

${ }^{18}$ Op. cit., p. 303-304.

${ }^{19}$ The Rise of the North Arabic script and its kur'änic development [Oriental Institute Publications, L]. Chicago, 1939; ce travail est complété et systématisé dans l'article "Arabic paleography" qui a déjà été mentionné. Dans une autre veine, mais toujours avec le souci d'organiser des listes de noms, l'approche adoptée par les auteurs du catalogue de l'exposition de Riyadh montre que l'obssession "nominaliste" est loin d'avoir disparu -malgré les difficultés évidentes à mettre les noms en rapport avec une écriture précise. Des 137 noms classés au début (op. cit., p. 27-28), une quinzaine seulement sont employés dans les notices descriptives des manuscrits; une trentaine viennent en complément.

${ }^{20}$ Voir les remarques très pertinentes de L. Volov, par ex.: "Flury's system was constructed for measuring the changes in writing, yet his object of study was less a form of writing than it was a mode of decoration" ("Plaited Kufic on Samanid epigraphic pottery", Ars Orientalis 6 [1966], 112).
} 
Et c'est bien là aussi l'une des difficultés que la paléographie des écritures livresques a rencontrées au cours de son développement: la calligraphie occupe une place importante dans la civilisation islamique et l'écriture n'est souvent appréhendée que par le biais de l'art de l'écriture ${ }^{21}$. Aussi n'est-il guère surprenant de constater que les contributions consacrées à la calligraphie arabo-islamique dépassent largement en nombre celles qu'a inspirées la paléographie des écritures livresques. Nous retrouvons d'ailleurs à ce point un problème déjà évoqué plus haut: la terminologie élaborée par les calligraphes ou par les milieux qui véhiculaient cette culture spécifique s'est imposée de facto à la paléographie.

Les difficultés que nous avons énumérées et qui ont émaillé l'histoire de la paléographie arabe des écritures livresques ne doivent pas toutefois retarder davantage les progrès en ce domaine. Un certain nombre de travaux préparatoires, réalisables sans trop de difficultés, sont la clé de tout progrès ultérieur. Le chantier qui mériterait la priorité est celui de la recherche des manuscrits datés: sur ce point, on ne peut que souscrire aux remarques formulées par F. Codera, plus que jamais d'actualité. En dépit de notre connaissance encore trop imparfaite des fonds de manuscrits arabes, cette enquête peut être menée à bien; elle a reçu une première forme de réalisation dans l'ouvrage de $\mathrm{K}$. 'Awwād qui répertorie les copies de textes antérieures à 500/1106 22 . Cette liste, qui répond avant tout à des préoccupations d'ordre philologique, a été compilée à partir de catalogues ou de publications d'inégale valeur, mais ne semble pas avoir été suivie d'un contrôle des manuscrits eux-mêmes: d'où des erreurs d'origines très diverses. Par la suite, d'autres recensements ont été menés à bien sur des bases plus spécifiques; on retiendra celui de G. Endress pour les débuts de l'écriture livresque ${ }^{23}$ ou encore celui que nous avons proposé pour les manuscrits $\mathrm{du} \mathrm{III}^{\mathrm{e}} / \mathrm{IX}^{\mathrm{e}}$ siècle $^{24}$. Sur une plus large assise, le Fichier des manuscrits moyen-orientaux datés (Fi.M.M.O.D.) a entrepris de publier des manuscrits datés antérieurs à 1500 , chaque reproduction étant accompagnée d'une description codicologique; à la fin 1997, 226 manuscrits avaient ainsi été décrits.

\footnotetext{
${ }^{21}$ Il est vrai que l'arabe hatt entretient une ambiguïté: bien que désignant en principe toute écriture, il évoque prioritairement la belle écriture. Le titre de l'exposition de Riyadh (voir n. 12), qui, en arabe, fait intervenir ce mot que la version anglaise rend par "calligraphy» est parfaitement illustratif de cette situation. Les publications en persan ou en turc évitent cette difficulté en réservant le mot hatt à la calligraphie et en utilisant un mot turc ou persan pour l'écriture non calligraphique.

${ }^{22}$ Aqdam al-mahtūtāt al-'arabiyya fímaktabāt al-"alam al-maktüba mundu sadr al-islām hattā sana 500h. (1106m.), Bagdad, 1982.

${ }^{23}$ Endress, G., "Handschriftenkunde", Handbuch der arabischen Philologie I, W. Fischer éd., Wiesbaden, 1982, p. 281.

${ }^{24}$ Déroche, F., "Les manuscrits arabes datés du III/IX $/{ }^{e}$ siècle», REI 55-57/1 (1987-89), 343-379.
} 
Ce travail est indispensable pour définir les contours chronologiques des différentes graphies. Le cas du magribi est de ce point de vue éloquent. Depuis l'article d'O. Houdas ${ }^{25}$, un certain accord s'était établi pour fixer l'apparition de cette écriture après le $\mathrm{IV}^{\mathrm{e}} / \mathrm{X}^{\mathrm{e}} \mathrm{s}$. Puis la découverte d'une copie du $k$. al-Muwatta' datée de 277/870 avait introduit un doute ${ }^{26}$; certaines publications suggéraient même que le magribī pouvait avoir été utilisé pour la copie des livres dès le $\mathrm{II}^{\mathrm{e}} / \mathrm{VIII}{ }^{\mathrm{e}} \mathrm{s}^{27}$. Comme l'a fait observer P. Sj. van Koningsveld ${ }^{28}$, l'emploi de papier et le contenu des gloses marginales sont des arguments décisifs pour reconsidérer la date de ce manuscrit. La question était à reprendre et la récente publication de deux fragments coraniques en caractère magrib $\bar{\imath}$ et de format oblong, datés l'un de 398/1008 et l'autre de $432 / 1040^{29}$, permet d'aboutir à des nouvelles propositions.

Cette quête opiniâtre servira à établir peu à peu une chronologie des écritures, laquelle constitue la base de toute analyse de l'évolution de la tradition manuscrite. Un article d'E. Whelan ${ }^{30}$ met en lumière les dangers d'une approche qui se fonde sur une documentation insuffisante et comporte de ce fait des lacunes fondamentales en matière de chronologie. L'auteur y compare en effect deux groupes de manuscrits comme s'ils étaient contemporains, alors qu'il ne dispose pas d'arguments sérieux pour établir qu'ils le sont effectivement ${ }^{31}$; les différences dont E.Whelan dresse la liste ${ }^{32}$ sont en partie le résultat d'une évolution sur un siècle et non pas le reflet des pratiques de deux groupes de copistes $^{33}$.

Le Fichier des manuscrits moyen-orientaux datés (Fi.M.M.O.D.) que nous évoquions plus haut se situe à mi-chemin entre le catalogue de manuscrits datés, tel qu'il s'est imposé dans le domaine de la paléographie latine, et l'album

\footnotetext{
${ }^{25}$ Houdas, O., "Essai sur l'écriture maghrébine», Nouveaux mélanges orientaux, Paris, 1886, p. 85-112.

${ }^{26}$ Ms Dublin, CBL 3001, Arberry, A. J., The Chester Beatty Library, A handlist of the Arabic manuscripts I. Dublin, 1955, p. 1.

${ }^{27}$ Abbott, N., The Rise..., p. 42, n. 192; James, D., Qur'ans and bindings from the Chester Beatty Library. Londres, 1980, p. 109.

${ }^{28}$ Op. cit., p. 29.

${ }^{29}$ Déroche, F., «Deux fragments coraniques maghrébins anciens au Musée des arts turc et islamique d'Istanbul", REI 59 (1991), 229-235.

${ }^{30}$ "Writing the word of God I", Ars Orientalis 20 (1990), 113-147.

${ }^{31}$ En fait, selon nos propres conclusions, le groupe 1 date du $\mathrm{II}_{\mathrm{\%}} \mathrm{IX}{ }^{\mathrm{e}} \mathrm{s}$. (voir Déroche, F., "The Qur'ān of Amājūr", MME 5 (1990-91), 59; The Abbasid tradition, Qur'ans of the 8th to the 10th centuries $A D$ [The Nasser D. Khalili collection of Islamic art I], Londres, 1992, p. 36-37), alors que le groupe 2 est antérieur. Mais il y aurait naturellement d'autres observations méthodologiques à faire. Ainsi, le groupe 2 ne comporte qu'un unique manuscrit, ce qui soulève la question de sa représentativité. Le choix même des deux groupes peut également être remis en cause; E. Whelan n'explique pas les raison qui l'ont amenée à retenir ces deux séries, alors que cette décision pèse lourdement sur les conclusions.

${ }^{32}$ Ibid., p. 123.

${ }^{33}$ Ibid., p. 123-124.
} 
traditionnel qui demeure un des outils essentiels dans l'étude des écritures livresques. A ce jour, plusieurs sont disponibles pour le chercheur $^{34}$ :

- Wright, W., Facsimiles of manuscripts and inscriptions (Oriental series), London, 1875-1883.

- Cheikho, L., Spécimens de cent écritures arabes pour la lecture des manuscrits anciens et modernes, Beyrouth, 1885.

- Moritz, B., Arabic palcography, A collection of Arabic texts from the first century of the hidjra till the year 1000, Cairo, 1905.

- Smith Lewis, A., and Dunlop Gibson, M., Forty-one facsimiles of dated Christian Arabic manuscripts, with text and English translation. With introductory observations on Arabic calligraphy by the Rev. D. S. Margoliouth [Studia sinaitica XII], Cambridge, 1907.

- Tisserant, E., Specimina codicum orientalium, Bonn, 1914.

- Arberry, A. J., India Office Library Specimens of Arabic and Persian palaography, London, 1939.

- Vajda, G., Album de paléographie arabe, Paris, 1958.

- Munajjid, S., Le manuscrit arabe jusqu'au X' de l'h., Le Caire, 1960.

- Witkam, J. J., Seven specimens of Arabic manuscripts preserved in the Library of the University of Leiden, Leiden, 1978.

- Fi.M.M.O.D. (Fichier des manuscrits moyen-orientaux datés), Paris, 1992-.

Ainsi que nous l'avons souligné précédemment, l'importance des comparaisons dans la démarche paléographique fait que les reproductions d'écritures, et donc les albums, représentent une ressource fondamentale pour le chercheur. Leur emploi est plus ou moins délicat: l'excellent ouvrage de W. Wright donne des références précises et décrit minutieusement chaque manuscrit. L'album de B. Moritz en revanche n'indique pas les cotes des ouvrages présentés, et les indications sont en général très pauvres ${ }^{35}$. Seul un nombre très réduit de facsimilés de manuscrits en écriture arabe a été publié à ce jour: ils ne peuvent jouer qu'un rôle très secondaire dans l'avancement des recherches. En tout état de cause, les reproductions aisément accessibles - dans des albums ou ailleurs-

\footnotetext{
${ }^{34}$ Pour mémoire, on peut signaler deux albums réalisés antérieurement à l'emploi des techniques de photographie: il s'agit des ouvrages de J. B. Silvestre (Paléographie universelle, Collection de facsimilés d'écriture de tous les peuples ... Première partie: Peuples orientaux, Paris, 1814) et de J. H. Möller (Paläographische Beiträge aus den herzoglichen Sammlungen in Gotha. 1. Heft. Erfurt, 1844). Nous ne signalons pas en revanche les livres qui concernent la calligraphie; ils peuvent être utiles au paléographe, mais leur parti pris les restreint à certains manuscrits.

${ }^{35}$ Le lecteur se reportera à la recension virulente de J. von Karabacek qui énumère les faiblesses de cet album ("Arabic Palæography», WZKM 20 [1906], 131-148).
} 
restent très peu nombreuses, ce qui contrarie cette démarche capitale pour la paléographie qu'est la constitution de séries de documents. Signalons que, dans la bibliographie de son livre La paléographie de l'Antiquité latine et du MoyenÂge occidental, B. Bischoff n'indiquait pas moins de 65 albums publiés au cours du même laps de temps (sans compter les séries de catalogues de manuscrits datés) $)^{36}$.

Les outils nécessaires, albums, mais aussi catalogues précis des collections, font donc encore souvent défaut. En outre, comme le soulignait J. J. Witkam ${ }^{37}$, la paléographie arabe souffre de l'absence d'une réflexion sur la façon dont il convient de procéder pour analyser et décrire l'écriture arabe. Jusqu'à présent, la façon de faire des paléographies occidentales a été reprise et adaptée à certaines spécificités de ce système alphabétique. Les épigraphistes ont été les premiers à suivre cette voie ${ }^{38}$-qui a été utilisée également pour les écritures livresques. La ressource essentielle consiste à dresser des tableaux qui présentent les formes des lettres de l'alphabet —ou de moins de certaines d'entre elles — afin de faciliter la comparaison d'une part et le travail de définition de l'autre.

Parmi tant d'autres exemples, l'évolution du käf final au cours des quatre premiers siècles montre bien que cette approche conserve tout son intérêt pour dater les manuscrits coraniques ${ }^{39}$. Dans un premier temps, sur les corans de style hiğgaz $\bar{l}$, la lettre se compose de trois éléments (Fig. 1a): un segment horizontal, posé sur la ligne de base, s'achève par une courbe "en U» qui se poursuit par un deuxième trait, légèrement orienté vers le haut et plus court que le premier; le dernier segment, à peu près perpendiculaire au précédent, se dirige vers la ligne supérieure qu'il effleure. À la fin du premier siècle, vraisemblablement dans le sillage de la réforme de 'Abd al-Malik, les deux traits horizontaux deviennent parallèles l'un à l'autre, celui du bas maintenant une longueur supérieure à celui du haut (Fig. 1b). Ce dessin est conservé au II $/ \mathrm{VIII}^{\mathrm{e}}$ s.: il est adapté aux nécessités de l'esthétique des écritures coraniques de la période et coexiste probablement avec un käf aux deux branches horizontales de même longueur (Fig. 1c). Ce dernier l'emporte totalement au $\mathrm{III}^{\mathrm{e}} / \mathrm{IX}^{\mathrm{e}} \mathrm{s}$. (Fig. 1d). Le changement de style qui marque la fin du III $/ \mathrm{IX}^{\mathrm{e}} \mathrm{s}$. et le début $\mathrm{du} \mathrm{IV} / \mathrm{X}^{\mathrm{e}} \mathrm{s}$. bouleverse une dernière fois les

\footnotetext{
${ }^{36}$ Bischoff, B., La paléographie de l'Antiquité latine et du Moyen-Âge occidental, Paris, 1985, p. 289-292.

${ }^{37}$ Loc. cit.

${ }^{38}$ Les exemples les plus caractéristiques sont ceux proposés par S. Fleury (voir par ex.: «Bandeaux ornementés à inscriptions arabes: Amida-Diarbekr. IX` siècle”, Syria 1 (1920), 234-249 et 318-328; "Le décor épigraphique des monuments de Ghazna", Syria 6 (1925), 61-90; "Le décor épigraphique des monuments fatimides du Caire», Syria 17 (1936), 365-376).

${ }^{39}$ «Un critère de datation des écritures coraniques anciennes, Le käf final ou isolé», Mélanges $M$. Meinecke, sous presse.
} 
habitudes: l'écriture coranique dominante, qui représente la version calligraphiée d'une "cursive", réintroduit un $k a \bar{f} f$ final dont la forme est apparentée à celle des corans hiğăzī (Fig. 1e).

Parallèlement, le module d'écriture devrait être pris en considération de manière plus systématique. Les sources soulignent l'importance de ce facteur; les études paléographiques n'en font pourtant qu'un usage très restreint. Nous avons eu l'occassion de démontrer le rôle de premier plan qu'il tenait au $\mathrm{III}^{\mathrm{e}} / \mathrm{IX}^{\mathrm{e}}$ s., un style se définissant entre autres choses par une certaine valeur du module de la ligne. Deux écritures contemporaines de l'époque, B II et D I, ont toutes deux été employées pour copier le Coran: mais alors que, dans la majorité des cas, BII apparaît sur des manuscrits comportant à la page 14,15 et 16 lignes de $c a .4$ à $c a$. $6 \mathrm{~mm}$ de haut en moyenne ${ }^{40}$, D I est présent sur des corans ayant $5,6,7,8$ ou 9 lignes à la page, pour une hauteur de ligne comprise entre $c a .11$ et $22 \mathrm{~mm}^{41}$.

L'approche évoquée ci-dessus a déjà permis d'aboutir à des résultats appréciables et continuera à occuper une place importante dans la mise au point des classements; il faut cependant constater qu'elle ne peut suffir à saisir toutes les caractéristiques de l'écriture arabe. Cela nous semble particulièrement évident en ce qui concerne la composante dynamique formée par les ligatures entre les lettres. Bien que faisant partie du système, elles n'apparaissent pas -et pour cause- dans les tableaux de lettres alors que leur réalisation par le copiste est loin d'être toujours identique ${ }^{42}$. Elles interviennent à l'intérieur du mot, sans toutefois que ce dernier corresponde automatiquement à une séquence de lettres liées entre elles: un mot peut en effet être formé d'une ou plusieurs de ces séquences dont la taille est variable (celles formées par trois graphèmes seraient les plus nombreuses ${ }^{43}$ ). Le corollaire de cette situation propre à l'écriture arabe est de mettre à la disposition du copiste deux types de "blancs" sur la ligne d'écriture, celui qui sépare un mot d'un autre et celui qui, le cas échéant, sépare les éléments constitutifs d'un même mot $^{44}$. Peut-être serait-il donc intéressant d'utiliser ces spécificités de l'écriture arabe pour en identifier les formes fondamentales, au-

${ }^{40}$ Déroche, F., "À propos d'une série de manuscrits coraniques anciens", Les manuscripts du Moyen-Orient, Essais de codicologie et de paléographie, F. Déroche éd., Paris-Istanbul, 1989, p. 103, Tableau II.

${ }^{41} I d$., "Qur'ān of Amājūr», p. 61 (voir aussi Chart I, p. 62).

${ }^{42}$ Il ne saurait être ici question de l'exploitation faite dans des compositions calligraphiques des possibilités plastiques des traits de ligature; ce genre de licence ne concerne pas vraiment les écritures livresques. Toutefois, les copistes des manuscrits peuvent jouer sur les possibilités d'élongation (mašq), de contraction ou de superposition.

${ }^{43}$ Renseignement communiqué par M. V. Atanasiu, que nous remercions.

44 À l'intérieur du mot, l'utilisation du ublanc» inclut également la façon d'organiser la séquence -en exploitant au besoin les possibilités de superposition et de chevauchement. 
delà des nombreuses variétés que tous lui reconnaissent et que l'étude des lettres prises individuellement permet de préciser.

Dans son livre déjà mentionné, B. Bischoff fait précéder son exposé sur l'histoire de l'écriture latine par la remarque suivante: "Il existe deux techniques de l'écriture fondamentalement différentes —qui ne sont toutefois assurément pas sans liens entre elles - je veux dire la calligraphie et la cursive. La première convient généralement aux écritures livresques; la seconde, à toute la gamme des écritures utilitaires. ${ }^{45}$ Cette distinction fondamentale entre des écritures "construites", où les lettres sont réalisées par une succession de traits de plume, et celles où «la lettre est, autant que possible, réalisée comme un tout, d'un seul trait de plume et est bientôt liée d'une manière convenable avec ses voisines ${ }^{46}$ ", n'est pas transposable telle quelle à l'écriture arabe. D'une part, les formes extrêmement simples de cette dernière n'exigent qu'un nombre très réduit de coups de calame. De l'autre, le système graphique repose dès les débuts sur des ligatures selon des règles bien différentes de celles qui régissent les cursives latines. Les critères qui se présentent d'emblée à l'esprit pour prendre en compte ces spécificités —plus ou moins grande rapidité d'exécution, soin apporté au dessin des lettres, décomposition ou enchaînement des gestes du copiste- font intervenir les uns et les autres une part de subjectivité qui risque de restreindre la validité de la démarche entreprise.

Il serait pourtant important de disposer de moyens pour faire le tri entre les différentes graphies qui apparaissent sur les manuscrits. Parmi ceux-ci, le paléographe rencontre aussi bien des copies soignées réalisées par un professionnel que des notes hâtivement jetées sur le papier et destinées à l'usage personnel; cette remarque ne reflète d'ailleurs nullement un point de vue moderne, si l'on en juge par les critiques qu'adressent certains auteurs médiévaux aux écritures peu soignées ${ }^{47}$. Comme ces dernières offrent des difficultés particulières pour l'analyse, il nous semble plus judicieux de faire porter, dans un premier temps, les efforts sur les écritures les plus régulières: aussi faut-il s'entendre sur ce qui les rend telles et nous nous proposons d'offrir quelques suggestions à ce propos. L'observation de la ligne de base que forment les ligatures entre les lettres pourrait venir appuyer les observations traditionnelles. A notre sens, une opposition entre les écritures à ligne de base rectiligne et celles où son parcours est animé par des saillants ou des rentrants viendrait clarifier le classement. Les

${ }^{45}$ Op. cit., p. 59.

${ }^{46}$ Op. cit., p. 60.

47 Voir par ex. Gacek, A., «Technical practices and recommendations recorded by classical and postclassical Arabic scholars concerning the copying and correction of manuscripts", Les manuscrits $d u$ Moyen-Orient, Essais de codicologie et de paléographie, F. Déroche éd., Paris-Istanbul, 1989, p. 55. 
écritures du premier ensemble pourraient être qualifiées de composées; par ce terme, nous entendons souligner le caractère apprêté du mouvement de la main exécutant une séquence de traits qui laissent transparaître aussi peu que possible - voire pas du tout- le geste du copiste.

Le deuxième ensemble ainsi défini correspondrait à des écritures "cursives", c'est-à-dire, dans le cas qui nous occupe, celles où le copiste limite considérablement les occasions de soulever son instrument et où le matériau subjectile - papyrus, parchemin ou papier - conserve la trace des mouvements de la main. En raison de cette particularité, nous proposons - pour échapper au mot "cursive» qui a déjà en paléographie un sens trop marqué- de risquer un néologisme et de parler d'écritures chirodictiques: il est indéniable que de nombreuses écritures livresques qui peuvent entrer dans cet ensemble sont le fruit d'un art très concerté. Mais à ce point, ce qui est véritablement décisif, c'est que la séquence "naturelle» des gestes n'a pas été substantiellement modifiée par l'effort calligraphique.

Un exemple permettra peut-être de clarifier ces propositions. La graphie D I dans notre système de classement des écritures coraniques anciennes appartient clairement à l'ensemble des composées: dans une séquence de lettres ligaturées, le trait demeure rectiligne (Fig. 2a). En revanche, le style NS I fait apparaître des interruptions dans la progression de la ligature, signe que nous nous trouvons en présence d'une écriture chirodictique (Fig. 2c), de même que dans le cas de l'écriture de style hịğā̌ $\bar{\imath}$ (Fig. 2b) ou dans celui d'une graphie plus récente (Fig. 2d-e).

Les deux ensembles, dont l'identification, permettrait de mieux cerner les grands axes de l'évolution de l'écriture arabe, doivent naturellement être à leur tour subdivisés. Ils se trouvent en effet compris entre deux pôles extrêmes: l'un (écritures calligraphiées ou soignées) réunit les graphies régulières qui nécessitent (ou s'imposent) un ductus en plusieurs étapes. De l'autre côté (écritures informelles), on retrouvera les écritures où, aux ligatures "obligatoires", s'ajoutent celles qui ont pour origine la commodité du copiste, les écritures marquées par des irrégularités non calculées dans le nombre des lignes, etc.

Pour tenter d'évaluer la position d'écritures d'un même style par rapport aux deux pôles évoqués ci-dessus, nous emprunterons un exemple au vaste ensemble des "cursives brisées" (que nous suggérons d'appeler écritures livresques abbassides). Le célèbre coran de la Nourrice ${ }^{48}$, copié en 410/1020 en Tunisie par 'Alī b. Aḥmad al-Warrāq, fait apparaître des nūn finaux dont l'exécution repose sur la décomposition du mouvement en trois temps (Fig. 3a). Peu de temps

\footnotetext{
${ }^{48}$ Ms Rakkada, Centre d'art islamique, [sans cote?]. Ce manuscrit a été fréquemment reproduit; nous mentionnons à titre indicatif Lings, M., The qur'änic art of calligraphy and illumination, London, 1978, pl. 10 et Itinéraire du savoir en Tunisie, Les temps forts de l'histoire tunisienne, Paris, 1995, p. 9.
} 
auparavant, à Palerme en $372 / 982-983^{49}$, un copiste anonyme avait transcrit le texte coranique dans une écriture du même groupe, mais de taille et de facture plus modestes; les trois segments de l'exemple précédent sont quelque peu estompés, bien que les contrastes entre graisses et maigres puissent encore être discernés (Fig. 3b). En 429/1039, à Kairouan cette fois, une section de la Mudawwana était copiée dans une version moins élaborée de ce même style ${ }^{50}$; le nūn y est réalisé encore plus rapidement, dans un geste qui, tout en respectant la forme générale de la lettre, ne marque plus les oppositions entre graisses et maigres et atténue très largement les différences les segments - dont en fait deux seulement subsistent (Fig. 3c). Ces trois formes de nün, toutes trois apparentées, montrent comment la même séquence théorique des déplacements de la main du copiste est susceptible de donner des résultats différents en fonction du soin plus ou moins grand qui est apporté au travail de copie. En forçant un peu l'interprétation, nous serions tenté de rapprocher la distinction que nous proposons de celle que A. Gacek analysait dans des textes mamlouks relatifs à la classification des écritures ${ }^{51}$.

Comme nous l'avons déjà souligné, et comme le suggérait déjà F. Codera, la paléographie des écritures livresques ne doit et ne peut progresser qu'avec l'appui de la codicologie. Une connaissance plus précise des techniques de fabrication des manuscrits dans le monde islamique permettra de multiplier les repères pour déterminer l'âge de copies non datées; et s'il est souhaitable que la paléographie des écritures livresques devienne un jour un moyen plus sûr de dater, il nous semble bon qu'elle le fasse dans le cadre d'un approche globale du livre manuscrit. Il appartient maintenant aux arabisants de se mettre à ce vaste chantier de manière à éviter qu'en 2098 un de nos successeurs puisse commencer le bilan de trois siècles d'études en redisant comme F. Codera: algo se ha hecho, pero poco.

\footnotetext{
${ }^{49}$ Ms Istanbul, Nuruosmaniye 23; quelques feuillets détachés se trouvent dans la Nasser D. Khalili collection of Islamic art (voir Déroche, F., Abbasid tradition, p. 146-151, n. ${ }^{\circ} 81$ ).

${ }^{50}$ Fehérvari, G., et Safadi, Y. H., 1400 years of Islamic art, A descriptive catalogue, London, 1981, p. 38-39, n. ${ }^{\circ}$ 10. Les auteurs suggèrent que le manuscrit a été copié en Andalus; il nous semble qu'il s'agit plutôt d'un des nombreaux manuscrits juridiques malikites du fond de la Grande mosquée de Kairouan (voir al-Buhlī al-Niyyāl, M., al-Maktaba al-a țariyya bi-l-Qayrawān, Tunis, 1963; Schacht, J., "On some manuscripts in the libraries of Kairouan and Tunis", Arabica 14 [1967], 225-258).

${ }^{51}$ "Arabic scripts and their characteristics as seen through the eyes of Mamluk authors", MME 4 (1989), 145.
} 


\section{RESUMÉ}

En 1898, Francisco Codera dressait un bilan sévère de l'état de la paléograpahie des écritures livresques arabes, mais proposait des moyens de faire avancer cette discipline. Un siècle plus tard, les progrès paraissent bien maigres. Quelles en sont les raisons? C'est, semble-t-il, l'histoire même de la paléographie qui peut expliquer les difficultés qu'elle a rencontrées. Pour l'avenir, une bonne partie du programme ébauché en 1898 est toujours d'actualité. Il convient également d'entamer une réflexion sur les méthodes d'analyse applicables à l'écriture arabe. Sur quelques points, des exemples sont proposés afin de lancer une plus vaste discussion.

\section{RESUMEN}

En 1898, Francisco Codera hacía un severo examen crítico del estado de la paleografía de la escritura árabe libresca, proponiendo no obstante los medios necesarios para hacer avanzar esta disciplina. Un siglo después, los progresos realizados parecen ser escasos. ¿A qué puede deberse esta situación? Diríase que la propia historia de la paleografía puede explicar las dificultades a que se ha enfrentado. Para el futuro, una gran parte del programa esbozado en 1898 sigue estando de actualidad. Conviene, asimismo, iniciar una reflexión sobre los métodos analíticos aplicables a la escritura árabe. Sobre algunos puntos concretos, se proponen en este artículo ejemplos que pueden servir de base a una discusión más amplia. 


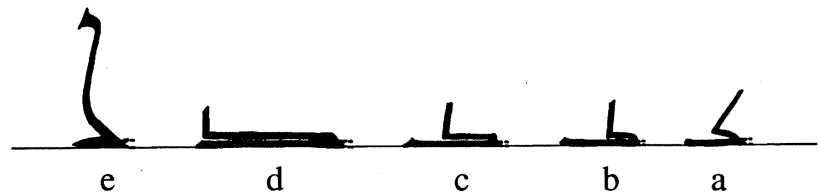

Pl. I: Formes du $k a \bar{f}$ final:

a) D'après le ms. Paris, BnF Arabe 328a (deuxième moitié du $I^{\mathrm{er}} / \mathrm{VII} \mathrm{e}^{\mathrm{e}} \mathrm{s}$.).

b) D'après un ms. Istanbul, Musée des arts turcs et islamique (inédit; fin du $\mathrm{I}^{\mathrm{er}} / \mathrm{début}$ du VII ${ }^{\mathrm{e}}$ siècle).

c) D'après le ms. Paris, BnF Arabe $334 \mathrm{~b}$ (II $/ \mathrm{VIII}^{\mathrm{e}}$ siècle).

d) D'après le ms. Paris, BnF Arabe $337 \mathrm{f}$ (II $/ \mathrm{IX}^{\mathrm{e}}$ siècle?).

e) D'après le ms. Téhéran, coll. Âqâ Mahdi Kâshânî (485 /1092).

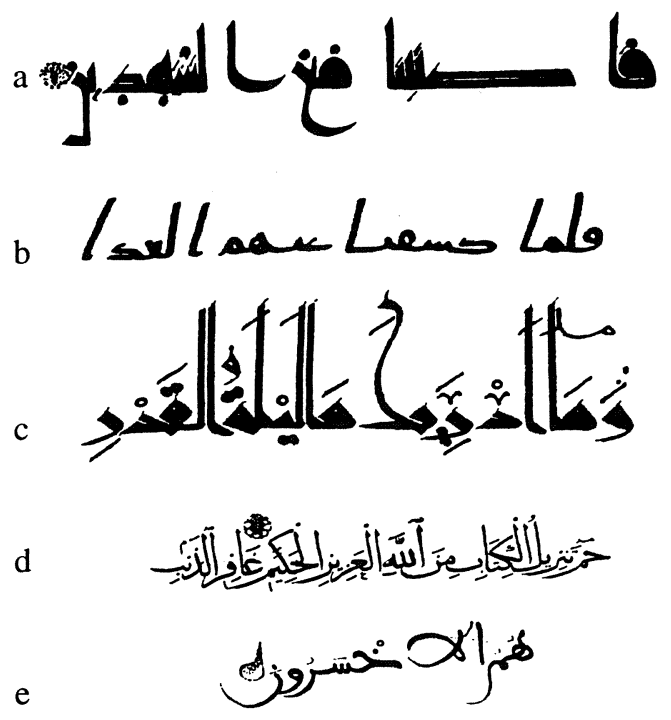

Pl. II: Écritures composée (a) et chirodictiques (b-e):

a) $\mathrm{D}$ 'après le ms. Paris, $\mathrm{BnF}$ Arabe $350 \mathrm{a}$ (III/IX $\mathrm{P}^{\mathrm{e}}$ siècle).

b) D'après le ms. Paris, BnF Arabe $328 \mathrm{a}$ (deuxième moitié du $\mathrm{I}^{\mathrm{e}} / \mathrm{VII}^{\mathrm{e}} \mathrm{s}$.).

c) D'après le ms. Téhéran, coll. Âqâa Mahdi Kâshânî (485/1092).

d) D'après le ms. Paris, BnF Arabe 5850 (entre 801 $/ 1399$ et $815 / 1412$ )

e) D'après le ms. Paris, BnF Arabe 440 (fin du IX $/ \mathrm{Xv}^{\mathrm{e}}$ siècle). 


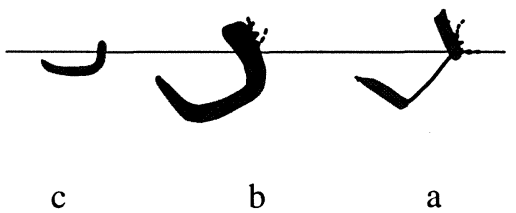

PI. III: Formes du nûn final:

a) Coran dit "de la Nourrice», d'après un fragment conservé à Raqqāda (410/1020).

b) D'après le ms. Istanbul, Nuruosmaniye 23 (372/982-983).

c) D'après le ms. Londres, Collection Khalili (429/1039). 